\title{
Impact of de novo vesicoureteral reflux on transurethral surgery outcomes in pediatric patients with ureteroceles
}

\author{
Sang Hoon Song ${ }^{1}$, Dong Hyun Lee ${ }^{1}$, Hwiwoo Kim ${ }^{1}$, Jongpil Lee', Sangmin Lee', Donghyun Ahn' , Sungchan Park ${ }^{2}$, \\ Kun Suk Kim ${ }^{1}$ \\ 'Department of Urology, Asan Medical Center, University of Ulsan College of Medicine, Seoul, ${ }^{2}$ Department of Urology, Ulsan University Hospital, University of Ulsan \\ College of Medicine, Ulsan, Korea
}

Purpose: We aimed to determine the impact of de novo vesicoureteral reflux (VUR) on postoperative urinary tract infection (UTI) and renal function in pediatric patients with ureteroceles.

Materials and Methods: We retrospectively reviewed the medical records of 34 patients (36 renal units) with ureteroceles that were treated endoscopically. Perioperative radiologic and clinical data regarding de novo VUR, UTI, and renal function were analyzed. Logistic regression analysis was used to identify factors that predicted unfavorable surgical outcomes, such as primary surgical failure, postoperative UTI, and deterioration of renal function.

Results: Of the 36 renal units, 22 had a duplex system (61.1\%). Preoperative VUR was noted in 9 units (25.0\%), including 3 units without renal duplication. Endoscopic surgery successfully decompressed the ureterocele and hydronephrosis in 28 units (77.8\%). De novo VUR developed in 18 renal units (50.0\%) postoperatively. The absence or presence of de novo VUR was not related to unfavorable surgical outcomes in univariate or multivariate analyses. Even after selection for the 28 renal units without preoperative VUR, the occurrence of de novo VUR had no predictive value for unfavorable surgical outcomes. Moreover, among the 14 renal units without renal duplication, de novo VUR had no predictive value for any of these adverse outcomes.

Conclusions: After endoscopic ureterocele puncture, de novo VUR is not significantly associated with postoperative UTI or deterioration in renal function in the long term. It may not, therefore, be necessary to reconstruct lower urinary tract routinely to correct de novo VUR after endoscopic puncture of the ureterocele.

Keywords: Endoscopy; Punctures; Ureterocele; Urinary tract infections; Vesico-ureteral reflux

This is an Open Access article distributed under the terms of the Creative Commons Attribution Non-Commercial License (http://creativecommons.org/licenses/by-nc/4.0) which permits unrestricted non-commercial use, distribution, and reproduction in any medium, provided the original work is properly cited.

\section{INTRODUCTION}

Obstructing ureteroceles in a single or duplicated renal system can trigger recurrent urinary tract infection
(UTI), vesicoureteral reflux (VUR), voiding dysfunction, and even deterioration of renal function. Various surgical options have been proposed to prevent these adverse events, including upper pole partial nephrectomy with or

Received: 17 November, 2018 - Accepted: 28 April, 2019

Corresponding Author: Kun Suk Kim

Department of Urology, Asan Medical Center, University of Ulsan College of Medicine, 88 Olympic-ro 43-gil, Songpa-gu, Seoul 05505, Korea

TEL: +82-2-3010-3740, FAX: +82-2-477-8928, E-mail: kskim2@amc.seoul.kr

ORCID: https://orcid.org/0000-0001-8982-6922 
without upper moiety ureterectomy and ureterocelectomy, lower moiety ureteral reimplantation, common sheath ureteral reimplantation, and transurethral incision and decompression of the ureterocele [1]. However, the best management strategy is still unknown owing to a lack of high-powered, randomized controlled trials comparing different surgical options.

Endoscopic puncture or incision of the ureterocele is the easiest and least invasive approach to managing ureteroceles in the pediatric population. The endoscopic approach seems to be the surgery of choice for selected patients, including those with intravesical ureteroceles [2-4]. Meanwhile, some studies have reported a low success rate of this approach with a high risk for subsequent procedures [3,5,6]. In these reports, concerns about the endoscopic puncture technique focus mainly on the high incidence of de novo VUR, up to $50 \%$ in the punctured moiety [7,8]. It has been noted that preoperative and postoperative VUR are related to trigonal anatomical distortion, which frequently mandates secondary surgery [9,10]. Some researchers insist that endoscopic surgery is unnecessary for preventing UTI [11]. Although modified techniques, such as fulguration of the internal mucosa of the ureterocele and the watering can procedure, have been introduced, the best method for preventing de novo VUR or UTI is still unknown, as is the causative relationship between de novo VUR and renal functional deterioration in the long term $[12,13]$.

In patients with duplex systems, additional surgery could be saved only for those patients with symptoms, regardless of VUR status or upper pole function after successful decompression of a dilated upper urinary tract [14]. However, the necessity and clinical value of additional surgery in asymptomatic patients with de novo VUR has been poorly evaluated until recently. Therefore, this study aimed to determine the impact of de novo VUR on postoperative UTI and renal function in pediatric patients with ureteroceles.

\section{MATERIALS AND METHODS}

The medical records of all pediatric patients with a single or duplex system who underwent primary endoscopic puncture of an ureterocele by two surgeons at a tertiary referral center from 1995 to 2013 were reviewed retrospectively. The report was prepared following the guidelines of the Institutional Review Board of Asan Medical Center (approval number: 2015-0473). Ureteroceles were categorized as intravesical or ectopic according to inspection by the surgeon at the time of cystoscopy. Patient demographics, thorough medical history, presence and degree of hydronephrosis on ultrasound by Society for Fetal Urology (SFU) grade, differential renal function on diethylenetriaminepentaacetic acid renal scan, and presence of VUR on voiding cystourethrography (VCUG) in the preoperative and postoperative periods were evaluated in all patients.

The indications for endoscopic puncture were a huge ureterocele shown to obstruct the bladder outlet on VCUG, increasing hydronephrosis on serial ultrasound follow-up, or febrile UTI. Endoscopic puncture was performed by two different techniques according to the surgeon's preference: large, single punctures and multiple, small punctures (Fig. 1). Each procedure was performed through an $8 \mathrm{Fr}$ pediatric cystoscope (Richard Wolf GmbH, Knittlingen, Germany) with Bugbee electrodes. For a large, single puncture, a hole through which an $8 \mathrm{Fr}$ cystoscope could easily pass was made with a 5 Fr Bugbee electrode at the intravesical, inferomedial portion of the ureterocele just above the bladder neck. In the second method, small, multiple punctures through which an 8 Fr cystoscope could not easily pass were made at two or more sites using a 2 Fr Bugbee electrode at the inferomedial portion of the ureterocele until the ureterocele decompressed securely.

The primary endpoint of this study was to evaluate the

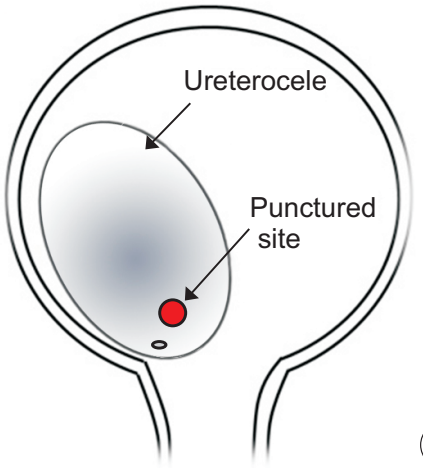

(A)

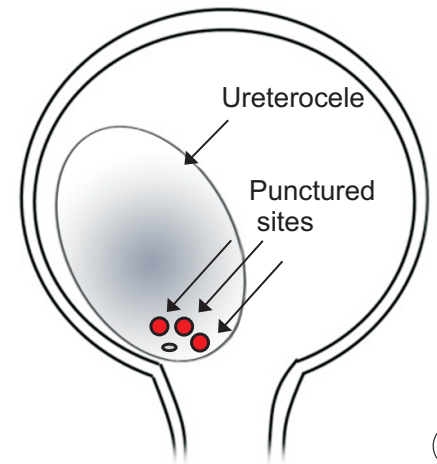

(B)
Fig. 1. Schematic images of the endoscopic ureterocele puncture technique. (A) A large, single puncture was made with a $5 \mathrm{Fr}$ Bugbee electrode at the intravesical, inferomedial portion of the ureterocele just above the bladder neck. (B) Small, multiple punctures were made at two or more sites using a $2 \mathrm{Fr}$ Bugbee electrode at the inferomedial portion of the ureterocele until the ureterocele decompressed securely. 
impact of de novo VUR on the occurrence of postoperative UTI and renal functional deterioration. All patients underwent renal and bladder ultrasounds at a median of 6 months after endoscopic treatment. VCUG and renal scan were performed 6 to 12 months after surgery or after febrile UTI. Decompression of hydronephrosis was defined as a downgrade of SFU grade in postoperative ultrasonography compared with the preoperative radiologic evaluation. $D e$ novo VUR was defined as newly developed VUR after the operation in the punctured moiety without preoperative reflux of the treated urinary system. The grade of VUR was classified according to the International Reflux Study grading system [15]. All radiologic studies were reviewed by pediatric radiologists at our institution. Primary success was defined as successful decompression of hydronephrosis after the procedure without the need for secondary interventions such as ureteral reimplantation, heminephrectomy, or repeat transurethral puncture. Long-term morbidity with UTI and renal functional deterioration were also evaluated.
Postoperative UTI was defined as a fever $>38.0^{\circ} \mathrm{C}$ with pyuria in urinalysis and positive urine culture or symptoms suggestive of a UTI confirmed by urine culture. Renal function was determined by MAG3 or dimercaptosuccinic acid renal scan. Renal functional deterioration was defined as a relative renal functional loss of more than $5 \%$ after the endoscopic surgery.

Data are presented as frequencies and percentages for categorical variables and as medians and interquartile ranges for continuous variables. Comparisons between groups were performed by using the chi-square or Fisher's exact test and linear-by-linear association for qualitative variables and the Mann-Whitney test for quantitative variables. Binary logistic regression analysis was used to identify factors that predicted unfavorable surgical outcomes, such as primary surgical failure, postoperative UTI, or deterioration of renal function. All reported p-values are two-sided, and $\mathrm{p}<0.05$ was considered statistically significant. Statistical analyses were performed using PASW

Table 1. Preoperative clinical parameters

\begin{tabular}{|c|c|c|c|c|}
\hline Parameter & Total & Single system & Duplication & p-value \\
\hline Number of renal units & $36(100.0)$ & $14(38.9)$ & $22(61.1)$ & \\
\hline Age (mo) & $3(1-11)$ & $7(1-85.2)$ & $2(1-5.2)$ & 0.192 \\
\hline Sex (male:female) & $9: 27$ & $7: 7$ & $2: 20$ & 0.014 \\
\hline Side (right:left) & 19:17 & $8: 6$ & $11: 11$ & 0.742 \\
\hline Prenatal diagnosis & $27(75.0)$ & $20(74.1)$ & $7(31.8)$ & 0.111 \\
\hline Presentation & & & & 0.378 \\
\hline Antenatal diagnosis & $20(55.6)$ & $7(50.0)$ & $13(59.1)$ & \\
\hline Urinary tract infection & $12(33.3)$ & $4(28.6)$ & $8(36.4)$ & \\
\hline Voiding difficulty & $4(11.1)$ & $3(21.4)$ & $1(4.5)$ & \\
\hline Size $>1 / 3$ of bladder & $18(50.0)$ & $6(42.9)$ & $12(54.5)$ & 0.733 \\
\hline Extent of ureterocele & & & & 0.054 \\
\hline Intravesical & $26(72.2)$ & $13(92.9)$ & $13(59.1)$ & \\
\hline Extravesical & $10(27.8)$ & $1(7.1)$ & $9(40.9)$ & \\
\hline Preoperative hydronephrosis (SFU grade) & & & & $<0.001$ \\
\hline 0 & $1(2.8)$ & $1(7.1)$ & $0(0.0)$ & \\
\hline 1 & $2(5.6)$ & $1(7.1)$ & $1(4.5)$ & \\
\hline 2 & $4(11.1)$ & $3(21.4)$ & $1(4.5)$ & \\
\hline 3 & $7(19.4)$ & $6(42.9)$ & $1(4.5)$ & \\
\hline 4 & $22(61.1)$ & $3(21.4)$ & $19(86.4)$ & \\
\hline \multicolumn{5}{|l|}{ Preoperative VUR } \\
\hline None & $27(75.0)$ & $11(78.6)$ & $16(72.7)$ & 1.000 \\
\hline Single-system ipsilateral VUR & $1(2.8)$ & $1(7.1)$ & - & \\
\hline Duplication ipsilateral upper moiety VUR & $1(2.8)$ & - & $1(4.5)$ & \\
\hline Duplication ipsilateral lower moiety VUR & $4(11.1)$ & - & $4(18.2)$ & \\
\hline Contralateral VUR & $4(11.1)$ & $2(14.3)$ & $2(9.1)$ & 0.634 \\
\hline Preoperative renal function & $47.1(37.0-50.1)$ & $48.4(21.8-49.8)$ & $44.6(37.0-51.0)$ & 0.716 \\
\hline
\end{tabular}

Values are presented as number (\%), median (interquartile range), or number only.

SFU, Society for Fetal Urology; VUR, vesicoureteral reflux. 
Statistics ver. 18.0 (IBM Corp., Armonk, NY, USA).

\section{RESULTS}

A total of 36 renal units (34 patients) underwent primary endoscopic puncture of the ureterocele at our hospital during the study period. Of these renal units, 27 (75.0\%) presented prenatally and $9(25.0 \%)$ presented postnatally. The patients' median age at the time of operation was 3 months, and the median follow-up period was 36 months. The study population consisted of 27 females and 9 males. Twenty-two patients (64.7\%) had a duplex system. Preoperative VUR was noted in 9 units $(25.0 \%)$, including 3 patients without renal duplication (Table 1). Female sex was dominant in the duplication group (90.9\%) compared with the singlesystem group (50.0\%). The incidence of any preoperative VUR to the ipsilateral or contralateral renal unit was not significantly different between the groups with a single system or a duplex system $(21.4 \%$ vs. $27.3 \%, \mathrm{p}=1.000)$. The duplication group showed more extravesical ureterocele than did the single-system group. However, the incidence of preoperative VUR was not significantly different between the intravesical and ectopic ureterocele groups (11.8\% vs. $26.3 \%, \mathrm{p}=0.451$ ).

Voiding dysfunction was the initial presentation of ureterocele in four patients. All of those patients were boys; they underwent ureterocele puncture at the age of 92,79 , 83, and 2 months, respectively. Three older children suffered from weak stream, straining, and frequency, whereas one infant showed oliguria and irritability as a chief complaint. All three children were free of symptoms immediately after the operation. One infant suffered one more episode of voiding difficulty but improved after transient urethral catheterization and was symptom-free afterward.

Clinical outcomes between the groups with a single or duplication system, such as postoperative UTI, secondary operation, and renal functional deterioration rate, were comparable (Table 2). However, the success rate was significantly higher in the single-system group, which could be explained by the higher rate of secondary operation in the duplication group. The incidence of any postoperative VUR overall was not significantly different between the single-system and duplication-system groups $(50.0 \%$ vs. $77.3 \%$, $\mathrm{p}=0.148)$. De novo VUR developed in 18 renal units $(50.0 \%)$

Table 2. Clinical outcomes in patients with single and duplicated systems

\begin{tabular}{|c|c|c|c|c|}
\hline Parameter & Total & Single system & Duplication & p-value \\
\hline Number of renal units & $36(100.0)$ & $14(38.9)$ & $22(61.1)$ & \\
\hline Follow-up periods (mo) & $36(19.25-66.50)$ & $33.5(16.7-48.2)$ & $50.5(18.5-89.5)$ & 0.236 \\
\hline Decompression of hydronephrosis & $28(77.8)$ & $13(92.9)$ & $15(68.2)$ & 0.115 \\
\hline Postoperative hydronephrosis (SFU grade) & & & & 0.487 \\
\hline 0 & $19(52.8)$ & $9(64.3)$ & $10(45.5)$ & \\
\hline 1 & $5(13.9)$ & $2(14.3)$ & $3(13.6)$ & \\
\hline 2 & $5(13.9)$ & $2(14.3)$ & $3(13.6)$ & \\
\hline 3 & $0(0.0)$ & $0(0.0)$ & $0(0.0)$ & \\
\hline 4 & $7(19.4)$ & $1(7.1)$ & $6(27.3)$ & \\
\hline \multicolumn{5}{|l|}{ Postoperative VUR } \\
\hline None & $12(33.3)$ & $7(50.0)$ & $5(22.7)$ & 0.148 \\
\hline Single-system ipsilateral VUR & $6(16.7)$ & $6(42.9)$ & - & \\
\hline Duplication, ipsilateral upper moiety VUR & $14(38.9)$ & - & $14(63.6)$ & \\
\hline Duplication, ipsilateral lower moiety VUR & $6(16.7)$ & - & $6(27.3)$ & \\
\hline Contralateral & $4(11.1)$ & $2(14.3)$ & $2(9.1)$ & \\
\hline De novo VUR & $18(50.0)$ & $5(35.7)$ & $13(59.1)$ & 0.305 \\
\hline Newly appeared lower moiety VUR & $2(5.6)$ & - & $2(9.1)$ & \\
\hline Persisting lower & $4(11.0)$ & - & $4(18.2)$ & \\
\hline Postoperative relative renal function & $45.4(38.1-50.0)$ & $44.6(35.7-51.0)$ & $47.3(46.3-50.0)$ & 0.421 \\
\hline Postoperative UTI & $15(41.7)$ & $3(21.4)$ & $12(54.5)$ & 0.083 \\
\hline Secondary operation & $11(30.6)$ & $2(14.3)$ & $9(40.9)$ & 0.142 \\
\hline Primary success & $22(61.1)$ & $12(85.7)$ & $10(45.5)$ & 0.033 \\
\hline Renal functional deterioration & $4(11.1)$ & $0(0.0)$ & $4(18.2)$ & 0.141 \\
\hline
\end{tabular}

Values are presented as number (\%) or median (interquartile range).

SFU, Society for Fetal Urology; UTI, urinary tract infection; VUR, vesicoureteral reflux. 
postoperatively. Meanwhile, newly appeared lower moiety VUR developed in 2 renal units (5.6\%). Postoperative UTI developed more frequently in patients with a duplication system (54.5\%) than in those with a single system (21.4\%), but this difference was not statistically significant $(\mathrm{p}=0.083)$. Four patients (11.1\%) showed a decrease in renal function as shown on the renal scan after endoscopic surgery.

Although de novo VUR occurred more frequently in the single, large puncture group than in the multiple, small puncture group (55.6\% vs. $11.1 \%$, $\mathrm{p}=0.026)$, de novo VUR was not related with unfavorable surgical outcomes, such as primary surgical failure, postoperative UTI, secondary operation, or deterioration of renal function in univariate and multivariate analyses (Table 3). The incidence of any postoperative VUR including contralateral VUR and lower moiety VUR (persisting or newly appeared postoperatively) was also not related with unfavorable surgical outcomes. Even after selecting for the 27 renal units without preoperative VUR, the rate of postoperative UTI was high but similar between cases with and without de novo VUR (40.0\% vs. 41.7\%). The occurrence of de novo VUR had no predictive value for primary success, secondary operation, or renal functional deterioration in renal units without preoperative VUR. Moreover, among the 14 renal units with a single system, de novo VUR had no predictive value for any of these adverse outcomes. By contrast, among the
22 renal units with renal duplication, a total of 9 renal units did require further interventions for insufficient ureterocele decompression or VUR. The success rate was slightly higher in the intravesical ureterocele group than in the ectopic group, but this difference was not statistically significant $(64.7 \%$ vs. $57.9 \%, \mathrm{p}=0.742)$. Of the 14 renal units with a single system, 5 ectopic ureters were all successfully treated with ureterocele decompression without the need for additional surgery. Three renal units required re-puncture for persistent dilatation of the ureterocele, while two renal units underwent successive reimplantation due to recurrent UTI or high-grade VUR, as shown in Table 4.

In binary logistic regression analyses, renal duplication was the only significant factor negatively associated with the primary success of endoscopic surgery. However, postoperative UTI, secondary operation, and renal functional deterioration were not related with renal duplication (Table 3).

\section{DISCUSSION}

Optimal ureterocele management remains a subject of debate, as it is difficult to directly compare and standardize the diverse surgical options in these cases that vary from minimally invasive endoscopic treatment to complicated major surgery [1]. Nevertheless, endoscopic surgery is widely

Table 3. Univariate logistic regression analysis of surgical outcomes in the study patients

\begin{tabular}{|c|c|c|c|c|c|c|c|c|}
\hline & \multicolumn{2}{|c|}{ Primary success } & \multicolumn{2}{|c|}{ Preop UTI } & \multicolumn{2}{|c|}{ Secondary operation } & \multicolumn{2}{|c|}{ Renal function decrease } \\
\hline & OR & p-value & OR & p-value & OR & p-value & OR & p-value \\
\hline Duplication & 0.139 & 0.024 & 4.400 & 0.057 & 4.154 & 0.105 & $3.590 \mathrm{E} 8$ & 0.999 \\
\hline Preop VUR & 1.048 & 0.764 & 1.164 & 0.845 & 0.213 & 0.172 & 0.000 & 0.999 \\
\hline Preop contralateral VUR & 2.053 & 0.552 & 0.429 & 0.483 & 0.733 & 0.799 & 0.000 & 0.999 \\
\hline Puncture technique & 2.800 & 0.248 & 0.625 & 0.560 & 0.571 & 0.534 & 0.000 & 0.999 \\
\hline De novo VUR & 1.000 & 1.000 & 0.795 & 0.735 & 1.300 & 0.718 & 3.400 & 0.311 \\
\hline Newly appeared lower moiety VUR & 1.222 & 0.892 & 0.818 & 0.892 & 1.500 & 0.785 & 0.000 & 0.999 \\
\hline
\end{tabular}

UTI, urinary tract infection; OR, odds ratio; preop, preoperative; VUR, vesicoureteral reflux.

Table 4. Indications for secondary surgery and the selected surgical methods after endoscopic puncture of ureterocele

\begin{tabular}{llc}
\multicolumn{1}{c}{ Indication for secondary surgery } & \multicolumn{1}{c}{ Surgical method } & \multicolumn{1}{c}{$\begin{array}{c}\text { Number of } \\
\text { renal units }\end{array}$} \\
\hline High-grade VUR and recurrent UTIs & Reimplantation & 1 \\
& Bulking agent injection and reimplantation & 1 \\
Not decompressed ureterocele with recurrent UTI & Re-puncture and reimplantation & 1 \\
and decreased renal function & & 1 \\
Not decompressed ureterocele & Re-puncture & 1 \\
Not decompressed ureterocele with nonfunctioning & Re-puncture and laparoscopic heminephrectomy and \\
upper moiety, recurrent UTI and high-grade VUR & reimplantation & 2 \\
Nonfunctioning upper moiety and recurrent UTI & Upper and lower urinary tract surgery & \\
\hline
\end{tabular}

VUR, vesicoureteral reflux; UTI, urinary tract infection. 
used as an initial treatment, because it is the least invasive procedure and is a definitive treatment to decompress obstructive ureteroceles and prevent unfavorable sequelae, such as UTI [16]. The main weakness of endoscopic surgery is the possibility of reoperation or secondary operation, such as ureteral reimplantation or hemi-nephrectomy of the upper moiety in a duplex system [17,18]. The best method for preventing de novo VUR or a subsequent operation is currently unknown. Moreover, there has been minimal research on the causative relationship between de novo VUR and renal functional deterioration over the long term $[12,13]$. Therefore, it is noteworthy that in our present study, de novo VUR after endoscopic surgery was not found to be related to primary success, postoperative UTI, secondary operation, or renal functional deterioration.

VUR occurs in $50 \%$ to $75 \%$ of patients with ureteroceles in duplicated renal systems, either primarily or secondary to endoscopic therapy $[1,13]$. The high incidence of de novo VUR causes many urologists to correct the VUR for fear of consequences such as a decrease in renal function [10,19]. However, Di Renzo et al. [4] found that de novo VUR resulted in subsequent surgery in only $11 \%$ of the patients in their study. Castagnetti et al. [20] reported a VUR incidence rate of $32 \%$ but a reoperation rate of only $5 \%$. Furthermore, similar rates of spontaneous VUR resolution after endoscopic puncture have been reported by multiple groups (40\%-59\%) [21-23]. Adorisio et al. [2] reported spontaneous resolution in 13 of 19 refluxing units (68\%) in 46 patients with a ureterocele in a duplex system. Of the 27 renal units without preoperative VUR in our current study, 15 (55.6\%) developed de novo VUR. Among these 15 renal units, 9 (60.0\%) did not need a secondary operation, which was not significantly different from the secondary operation rate (66.7\%) in the 12 patients in our current series without de novo VUR. Moreover, the occurrence of de novo VUR had no predictive value for postoperative febrile UTI or renal functional deterioration.

Preoperative VCUG is essential for evaluating the anatomy of the ureterocele, bladder morphology, and possible bladder outlet obstruction during voiding. Preoperative VCUG is also necessary to identify any VUR and to decide whether to start antibiotic prophylaxis if needed, even in patients with a single-system ureterocele. According to the present study, however, postoperative VCUG might be spared in those who develop postoperative febrile UTI. This result is consistent with previous reports suggesting that de novo VUR can be managed without treatment in a significant number of cases [4]. Therefore, a hasty decision to perform lower tract reconstruction in patients with a ureterocele in a duplex system with associated VUR should be avoided. Nevertheless, the intention of this study is not to recommend that endoscopic surgery is the universal remedy for patients with ureterocele. Rather, we claim that judicious use of not only endoscopic surgery but also reconstructive surgery is necessary for these patients.

One of the remarkable results of our present study was that the postoperative rate of UTI in patients without preoperative VUR was high but similar between cases with and without de novo VUR (40.0\% vs. 41.7\%). The postoperative rate of UTI was not significantly different between patients with and without a preoperative UTI history (60.0\% vs. $34.6 \%$, $\mathrm{p}=0.260$ ). Of the five patients in our current series who had a postoperative UTI without de novo VUR, three cases had symptomatic cystitis with urinary frequency or dysuria without fever. Endoscopic puncture of the ureterocele often results in a large ureterocele flap volume in situ. Although bladder dysfunction associated with ureteroceles remains unclear, some studies have suggested that a muscular defect at the bladder neck and trigonal anatomical distortion might cause bladder dysfunction [24-26]. Therefore, cystitis in patients with a decompressed ureterocele in situ is not unexpected.

We showed that the success rate was slightly higher in the intravesical ureterocele group than in the ectopic group, but this difference was not statistically significant $(64.7 \%$ vs. $57.9 \%, \mathrm{p}=0.742$ ). Sander et al. [27] similarly reported that patients with ectopic ureteroceles showed a higher rate of secondary surgery than did patients with intravesical ureteroceles ( $80.8 \%$ vs. $67.7 \%$, $\mathrm{p}=0.3681$ ). In their cohort, there were no patients with extravesical single-system ureters. By contrast, five ectopic ureters in a single system among our cohort showed a successful outcome. In a meta-analysis reported by Byun and Merguerian [9], the reoperation rate was significantly higher in patients with ectopic ureteroceles after a pooled analysis.

We compared two different endoscopic puncture techniques in the present study. The clinical outcomes, such as postoperative UTI, secondary operation, and renal functional deterioration rate, were comparable between the two techniques. At first, we hypothesized that the multiple, small puncture technique might lower the possibility of de novo VUR compared with the single, large puncture technique, accomplishing successful decompression of the ureterocele and decreasing the incidence of secondary operations and renal functional deterioration. As expected, the rate of de novo VUR was found to be lower in our multiple, small puncture group. However, this was not associated with subsequent surgery or poor renal function. Similarly, Palmer 
et al. [13] compared the incision technique and the puncture technique using a holmium laser to make 10 to 20 holes. They concluded that the multiple-puncture technique results in a decreased incidence of de novo VUR, consistent with our current result. However, those authors also reported fewer secondary invasive procedures after endoscopic surgery with the multiple-puncture technique than with the single-incision technique. Indications for subsequent anti-reflux surgery in the study of Palmer and colleagues were de novo VUR in the ureterocele moiety, the ipsilateral moiety, and the contralateral renal unit. It is possible that the rate of secondary surgery would have been similar in the two groups in their study if VUR had been allowed to resolve spontaneously. Nevertheless, we agree that it is reasonable to make every effort to minimize de novo VUR at the time of ureterocele puncture, because the large puncture technique did not have benefit for the resolution rate of hydronephrosis but showed a lower rate of de novo VUR, although the clinical impact of de novo VUR was less than expected.

The limitations of our study included the nonrandomized and retrospective nature of the analysis, meaning that the size, location, and number of holes were not standardized between patients in each group. We used a Bugbee electrode to puncture the wall of the ureterocele. However, it is more difficult to make small holes with a $3 \mathrm{Fr}$ electrode than with a holmium laser. This difficulty might have increased the incidence of de novo VUR in our small, multiplepuncture group. Nevertheless, the rate of de novo VUR was lower in our multiple-puncture group than in our single, large puncture group. The small sample size was another limitation of our present analyses. Well-designed, randomized, prospective, multi-center studies are required in the future to determine the optimal management of obstructive ureteroceles.

\section{CONCLUSIONS}

The incidence of de novo VUR after endoscopic treatment of ureterocele is $50 \%$ overall and $59.1 \%$ in the duplicated system. However, de novo VUR is not significantly associated with postoperative UTI or renal functional deterioration in the long term. It may not, therefore, be necessary to perform lower urinary tract reconstruction routinely to correct de novo VUR after endoscopic puncture of the ureterocele.

\section{CONFLICTS OF INTEREST}

The authors have nothing to disclose.

\section{ACKNOWLEDGMENTS}

This manuscript was selected as the best paper at the 69th Korean Urological Association meeting in 2017.

\section{REFERENCES}

1. Castagnetti M, El-Ghoneimi A. Management of duplex system ureteroceles in neonates and infants. Nat Rev Urol 2009;6:30715.

2. Adorisio O, Elia A, Landi L, Taverna M, Malvasio V, Danti AD. Effectiveness of primary endoscopic incision in treatment of ectopic ureterocele associated with duplex system. Urology 2011;77:191-4

3. Boucher A, Cloutier J, Rousseau A, Charrois R, Bolduc S. Is an initial endoscopic treatment for all ureteroceles appropriate? J Pediatr Urol 2013;9:339-43.

4. Di Renzo D, Ellsworth PI, Caldamone AA, Chiesa PL. Transurethral puncture for ureterocele-which factors dictate outcomes? J Urol 2010;184(4 Suppl):1620-4.

5. Coplen DE, Barthold JS. Controversies in the management of ectopic ureteroceles. Urology 2000;56:665-8.

6. Shokeir AA, Nijman RJ. Ureterocele: an ongoing challenge in infancy and childhood. BJU Int 2002;90:777-83.

7. Hagg MJ, Mourachov PV, Snyder HM, Canning DA, Kennedy WA, Zderic SA, et al. The modern endoscopic approach to ureterocele. J Urol 2000;163:940-3.

8. Coplen DE, Duckett JW. The modern approach to ureteroceles. J Urol 1995;153:166-71.

9. Byun E, Merguerian PA. A meta-analysis of surgical practice patterns in the endoscopic management of ureteroceles. J Urol 2006;176(4 Pt 2):1871-7; discussion 1877.

10. Elder JS. Guidelines for consideration for surgical repair of vesicoureteral reflux. Curr Opin Urol 2000;10:579-85.

11. Blyth B, Passerini-Glazel G, Camuffo C, Snyder HM 3rd, Duckett JW. Endoscopic incision of ureteroceles: intravesical versus ectopic. J Urol 1993;149:556-9; discussion 560.

12. Kajbafzadeh A, Salmasi AH, Payabvash S, Arshadi H, Akbari HR, Moosavi S. Evolution of endoscopic management of ectopic ureterocele: a new approach. J Urol 2007;177:1118-23; discussion 1123.

13. Palmer BW, Greger H, Mannas DB, Kropp BP, Frimberger D. Comparison of endoscopic ureterocele decompression techniques. Preliminary experience--is the watering can puncture superior? J Urol 2011;186(4 Suppl):1700-3. 
14. Castagnetti M, Vidal E, Burei M, Zucchetta P, Murer L, Rigamonti W. Duplex system ureterocele in infants: should we reconsider the indications for secondary surgery after endoscopic puncture or partial nephrectomy? J Pediatr Urol 2013;9:11-6.

15. Report of the International Reflux Study Committee. Medical versus surgical treatment of primary vesicoureteral reflux: a prospective international reflux study in children. J Urol 1981;125:277-83.

16. Chertin B, Rabinowitz R, Pollack A, Koulikov D, Fridmans A, Hadas-Halpern I, et al. Does prenatal diagnosis influence the morbidity associated with left in situ nonfunctioning or poorly functioning renal moiety after endoscopic puncture of ureterocele? J Urol 2005;173:1349-52.

17. Ziylan O, Oktar T, Korgali E, Nane I, Alp T, Ander H. Lower urinary tract reconstruction in ectopic ureteroceles. Urol Int 2005;74:123-6.

18. de Jong TP, Dik P, Klijn AJ, Uiterwaal CS, van Gool JD. Ectopic ureterocele: results of open surgical therapy in 40 patients. J Urol 2000;164:2040-3; discussion 2043-4.

19. Jayanthi VR, Koff SA. Long-term outcome of transurethral puncture of ectopic ureteroceles: initial success and late problems. J Urol 1999;162(3 Pt 2):1077-80.

20. Castagnetti M, Cimador M, Sergio M, de Grazia E. Transurethral incision of duplex system ureteroceles in neonates: does it increase the need for secondary surgery in intravesical and ectopic cases? BJU Int 2004;93:1313-7.

21. Chertin B, de Caluwé D, Puri P. Is primary endoscopic puncture of ureterocele a long-term effective procedure? J Pediatr Surg 2003;38:116-9; discussion 116-9.

22. Chertin B, Fridmans A, Hadas-Halpren I, Farkas A. Endoscopic puncture of ureterocele as a minimally invasive and effective long-term procedure in children. Eur Urol 2001;39:332-6.

23. De Gennaro M, De Grazia E, Nappo S. [The prenatal diagnosis and early endoscopic section of ureterocele]. Pediatr Med Chir 1996;18:585-9. Italian.

24. Sherman ND, Stock JA, Hanna MK. Bladder dysfunction after bilateral ectopic ureterocele repair. J Urol 2003;170:1975-7.

25. Abrahamsson K, Hansson E, Sillén U, Hermansson G, Hjälmås K. Bladder dysfunction: an integral part of the ectopic ureterocele complex. J Urol 1998;160:1468-70.

26. Paye-Jaouen A, Pistolesi F, Botto N, Enezian G, GrapinDagorno C, Peycelon M, et al. Long-term bladder function after ureterocele decompression in children. J Urol 2015;193(5 Suppl):1754-9.

27. Sander JC, Bilgutay AN, Stanasel I, Koh CJ, Janzen N, Gonzales ET, et al. Outcomes of endoscopic incision for the treatment of ureterocele in children at a single institution. J Urol 2015;193:662-6. 